\title{
Effect of rubber polarity on selective wetting of carbon nanotubes in ternary blends
}

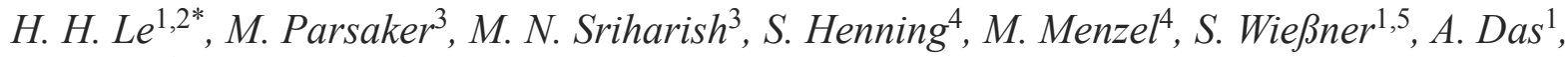 \\ Q. K. Do ${ }^{6}$, G. Heinrich ${ }^{1,5}$, H-J. Radusch ${ }^{7}$ \\ ${ }^{1}$ Leibniz-Institut für Polymerforschung Dresden e.V., D-01069 Dresden, Germany \\ ${ }^{2}$ Institut für Polymerwerkstoffe e.V., D-06217 Merseburg, Germany \\ ${ }^{3}$ Center of Engineering Sciences, Martin Luther University Halle-Wittenberg, D-06099 Halle/S., Germany \\ ${ }^{4}$ Fraunhofer IWM, D-06120 Halle/S., Germany \\ ${ }^{5}$ Technische Universität Dresden, Institut für Werkstoffwissenschaft, D-01062 Dresden, Germany \\ ${ }^{6}$ Institute of Chemistry, Vietnamese Academy of Science and Technology, Hanoi, Vietnam \\ ${ }^{7}$ Polymer Service GmbH Merseburg, D-06217 Merseburg, Germany
}

Received 1 April 2015; accepted in revised form 13 June 2015

\begin{abstract}
Based on atomic force microscopy (AFM) and Fourier transform infrared spectroscopy (FTIR) analysis of the rubber-filler gel (wetting concept) the kinetics of selective wetting of carbon nanotubes (CNTs) in ternary styrene butadiene rubber (SBR)/butadiene rubber (BR)/natural rubber (NR) blends was qualitatively and quantitatively characterized. Almost all CNTs are found to be wetted by the non-polar NR but not by the other non-polar rubber like BR or weakly polar SBR. It was proposed that phospholipids, which are linked to the $\alpha$-terminal of NR can interact with the CNT surface through cation- $\pi$ interactions forming strong bonding between NR and CNTs. Using the corrected surface tension value of NR, which involves the effect of phospholipids found in our previous work the selective wetting of CNTs in ternary rubber blends can be well predicted using the Z-model for a thermodynamic equilibrium state. By replacing the non-polar BR by a polar rubber like nitrile butadiene rubber (NBR) as a blend component CNTs are wetted by NBR slightly more than by NR thanks to the strong interaction between CNTs and nitrile groups of NBR. SBR remains unbound to CNTs in both blends.
\end{abstract}

Keywords: manocomposites, carbon nanotubes, rubber blends, selective filler wetting

\section{Introduction}

Carbon nanotubes (CNTs) with special nanoscale structure combined with the high strength of the carbon-carbon bondings and a large aspect ratio opens up a wide range of new applications [1]. However, the physical properties of the CNT-polymer composites are often below expectations, because a sufficiently effective force transfer between the polymer matrix and CNTs has not yet been reached. In order to exploit the exceptional mechanical and electrical properties of CNTs in polymer composites, the filler wetting and dispersion as well as the filler-polymer interaction must be increased [2-4]. It is generally known, that the smaller the contact angle $\theta$ between polymer and filler, the better the wettability of the filler and the stronger the polymer-filler interaction. Although the contact angle $\theta$ can be calculated from the surface tension of the individual components of the mixture, it is not sufficient for the description of the complex wetting behavior of the filler in rubber compounding [5]. The reason is that the filler wetting is not only thermodynamically determined, but it is also a kinetic process. Due to the agglomerate structure of filler polymer chains need a certain time

\footnotetext{
${ }^{*}$ Corresponding author, e-mail: le-haihong@ipfdd.de

(C) BME-PT
} 
to wet and infiltrate into the voids and pores of the agglomerates. In addition, the evaluation of filler wetting based on the contact angle $\theta$ is always associated with a large uncertainty, because the filler surface is often absorbed by other ingredients of the mixture during the mixing process, especially in rubber processing. That leads to a significant and uncontrolled change in the surface energy of the filler and thus its wettability [6]. Different methods [7-17] were developed and used for characterization of filler wetting and polymer-filler interaction in nanocomposites containing CNTs and single polymer. Scanning electron microscopy (SEM) of cryo-fractured surfaces of the composites was used for visualization of the polymer layer bonded to the filler $[7,8]$. Using dynamic mechanical analysis (DMTA) for filled rubber compounds a broad peak of the loss factor in the temperature range of the material glass transition temperature was observed and attributed to the reduced mobility of the bound polymer layer [11]. The shift of the D and G bands in the Raman spectra of the CNT composites was discussed as a result of the CNT-matrix wetting $[9,10]$ and cation- $\pi$ bonds between ionic liquid and CNTs [11]. Analysis of rubber-filler gel after extraction experiments (bound rubber measurement) by means of nuclear magnetic resonance (NMR), pyrolysis gas chromatography (PGC) and Fourier transform infrared spectroscopy (FTIR) as well as thermogravimetric analysis (TGA) was carried out for qualitative and quantitative characterization of the physical background of the filler-polymer interaction in carbon black or silica filled rubber compounds [12-17]. If the rubber matrix consists of two or more rubber components the wetting of filler aggregates becomes much inhomogeneous because of the different affinity of filler to rubber components. Up to date, the selective wetting of filler in rubber blends has not been characterized systematically so far. In our previous works, the selective localization of carbon black (CB) [18] and silica in binary [19] and ternary rubber blends [5] were thoroughly characterized by means of the wetting concept based on the TGA and FTIR analysis of rubber-filler gel. Recently, the selective localization of CNTs in binary styrene butadiene rubber (SBR)/natural rubber (NR) blends was investigated [20]. An unusually high loading of CNTs localized in the NR phase was found and the strong cation- $\pi$ interaction between the linked phospho- lipids of NR and the $\pi$ electrons of CNT surface was proposed to explain this result.

In the present work we modified the wetting concept in order to characterize the kinetics of selective wetting of CNT surface by rubber components of ternary blends based on SBR/BR/NR and SBR/NBR/ NR. The effect of strong cation- $\pi$ interaction of NR and CNTs as well as the effect of rubber polarity by replacement of BR by NBR on the selective wetting of CNTs will be characterized and discussed.

\section{Experimental}

\subsection{Materials and mixture preparation}

Solution styrene butadiene rubber (S-SBR) SPRINTAN SLR-4601 (Styron Deutschland GmbH) with a styrene content of $21 \%$ and vinyl content of $63 \%$, butadiene rubber (BR) Buna cis 132 (Styron Deutschland $\mathrm{GmbH}$ ) and nitrile butadiene rubber (NBR) Perbunan 3445F (Lanxess) with a nitrile content of $34 \%$ as well as natural rubber (NR) Standard Malaysian Rubber (SMR10) (Weber \& Schaer $\mathrm{GmbH}$ ) were used as rubber matrix. NR was masticated by means of two-roll mill before use in order to obtain a similar Mooney viscosity value as the other blend partners. Multi-walled carbon nanotubes (CNTs) Nanocyl ${ }^{\mathrm{TM}}$ NC7000 (Nanocyl S.A., Belgium) with an average diameter of $10 \mathrm{~nm}$ and a broad length distribution with several nanotubes up to $10 \mu \mathrm{m}$ were used as filler. An amount of peroxide (Peroxan DC, Pergan GmbH, Germany) of 1 phr was used as vulcanizing agent for all the mixtures.

For preparation of CNT filled compounds and blends an internal mixer (Rheocord 300p, ThermoHaake) was used by keeping the following mixing conditions: initial chamber temperature $T_{\mathrm{A}}$ of $50^{\circ} \mathrm{C}$, rotor speed of $75 \mathrm{rpm}$, fill factor of 0.68 . A conductivity sensor system was installed in the chamber of the internal mixer to measure the electrical signal of the conductive mixtures. CNT filled compounds were prepared by mixing $7 \mathrm{phr}$ CNTs with different rubbers investigated. Two ternary blends CNT-SBR/BR/ NR with a weight ratio of 33/33/34 and CNT-SBR/ NBR/NR with a weight ratio of 33/33/34 containing $5 \mathrm{phr}$ CNTs were prepared in one-step mixing process (statistical blends). For achieving ternary blends from masterbatch mixing a masterbatch CNT-SBR was prepared by pre-mixing CNTs in SBR and then the masterbatch was mixed with two other fresh rubbers in order to receive two masterbatch blends 
(CNT-SBR)/BR/NR and (CNT-SBR)/NBR/NR with the composition corresponding to that of the statistical blend. For an effective dispersion of CNTs in rubber matrix an ethanol-assisted mixing process (wet mixing) as described in our previous work [20] was applied. CNTs were first wetted with a certain amount ethanol to a paste. Then, it was added into the mixing chamber with rubber for preparation of the composites without any additional surfactant. Ethanol was entirely vaporized during the mixing process. A weight ratio ethanol/CNT of 3.7 was chosen for a good dispersion of CNTs. Samples were taken out during the mixing process at different times for further investigation.

\subsection{Determination of rubber layer $L$}

An amount of $0.1 \mathrm{~g}$ of each uncured mixture was stored in $100 \mathrm{~mL}$ toluene at room temperature for seven days. The solution was cast from the flask and the rubber-filler gel was taken out and dried up in an oven with a temperature of $70^{\circ} \mathrm{C}$ for $3 \mathrm{~h}$ to a constant mass. At least two experiments were carried out for each sample. Rubber layer $L$ was calculated using Equation (1) [18, 19]:

$L=\frac{m_{2}-m_{1} \cdot c_{\mathrm{CNT}}}{m_{2}}$

The mass $m_{1}$ is corresponding to the rubber compound before extracting. $m_{2}$ is the mass of the rubber-filler gel, which is the sum of the undissolvable rubber part and the mass of CNTs. $c_{\mathrm{CNT}}$ is the mass concentration of CNTs in the composite.

\subsection{Experimental determination of selective filler wetting in ternary blends}

Experimental determination of filler wetting by means of the wetting concept was carried out according to procedure described in details in our previous work for silica filled rubber blend [19] The fraction of filler surface $S^{\mathrm{B}(\mathrm{SBR})}(t), S^{\mathrm{B}(\mathrm{BR})}(t)$ and $S^{\mathrm{B}(\mathrm{NR})}(t)$ wetted by the SBR, BR and NR phase, respectively, in ternary SBR/BR/NR blend can be determined according to Equations (2)-(4).

$$
\begin{aligned}
& \frac{S^{\mathrm{B}(\mathrm{SBR})}(t)}{S^{\mathrm{B}(\mathrm{BR})}(t)}=\frac{L_{\mathrm{P}}^{\mathrm{BR}}}{L_{\mathrm{P}}^{\mathrm{SBR}}} \cdot \frac{L^{\mathrm{B}(\mathrm{SBR})}(t)}{L^{\mathrm{B}(\mathrm{BR})}(t)} \\
& \frac{S^{\mathrm{B}(\mathrm{BR})}(t)}{S^{\mathrm{B}(\mathrm{NR})}(t)}=\frac{L_{\mathrm{P}}^{\mathrm{NR}}}{L_{\mathrm{P}}^{\mathrm{BR}}} \cdot \frac{L^{\mathrm{B}(\mathrm{BR})}(t)}{L^{\mathrm{B}(\mathrm{NR})}(t)}
\end{aligned}
$$

$$
\begin{aligned}
S^{\mathrm{B}(\mathrm{SBR} / \mathrm{NBR} / \mathrm{NR})}(t) & = \\
S^{\mathrm{B}(\mathrm{SBR})}(t) & +S^{\mathrm{B}(\mathrm{BR})}(t)+S^{\mathrm{B}(\mathrm{NR})}(t)
\end{aligned}
$$

$S^{\mathrm{B}(\mathrm{SBR} / \mathrm{BR} / \mathrm{NR})}(t)$ is the total filler surface wetted by the blend at the mixing time $t$. The plateau values $L_{\mathrm{P}}^{\mathrm{SBR}}$, $L_{\mathrm{P}}^{\mathrm{BR}}$ and $L_{\mathrm{P}}^{\mathrm{NR}}$ are the rubber layer at the end of the wetting process of SBR, BR and NR in single compounds experimentally determined (see section 3.2). $L^{\mathrm{B}(\mathrm{SBR})}(t), L^{\mathrm{B}(\mathrm{BR})}(t)$ and $L^{\mathrm{B}(\mathrm{NR})}(t)$ are the rubber layer of the blend component SBR, NBR and NR at mixing time $t$, respectively. Their sum is the rubber layer $L^{\mathrm{B}(\mathrm{SBR} / \mathrm{BR} / \mathrm{NR})}(t)$, which is experimentally determined by the extraction experiment of the blend. Using FTIR analysis of the rubber-filler gel the values of $L^{\mathrm{B}(\mathrm{SBR})}(t), L^{\mathrm{B}(\mathrm{BR})}(t)$ and $L^{\mathrm{B}(\mathrm{NR})}(t)$ can be determined from $L^{\mathrm{B}(\mathrm{SBR} / \mathrm{NBR} / \mathrm{NR})}(t)$ and the ratio of peak area $A^{\mathrm{SBR}} / A^{\mathrm{BR}}$ and $A^{\mathrm{BR}} / A^{\mathrm{NR}}$ according to Equations (5)-(6) as described in our previous works [19]:

$$
\begin{aligned}
& \frac{L^{\mathrm{B}(\mathrm{SBR})}(t)}{L^{\mathrm{B}(\mathrm{BR})}(t)}=\frac{1}{f_{\mathrm{SBR} / \mathrm{BR}}} \cdot \frac{A^{\mathrm{SBR}}(t)}{A^{\mathrm{BR}}(t)} \\
& \frac{L^{\mathrm{B}(\mathrm{BR})}(t)}{L^{\mathrm{B}(\mathrm{NR})}(t)}=\frac{1}{f_{\mathrm{BR} / \mathrm{NR}}} \cdot \frac{A^{\mathrm{BR}}(t)}{A^{\mathrm{NR}}(t)}
\end{aligned}
$$

$A^{\mathrm{SBR}}, A^{\mathrm{BR}}$ and $A^{\mathrm{NR}}$ are the areas of a characteristic peak of the SBR phase at $1494 \mathrm{~cm}^{-1}$ (stretching vibration of the phenyl ring) and BR phase at $3004 \mathrm{~cm}^{-1}$ (stretching mode $\mathrm{C}-\mathrm{H}$ ) as well as NR phase at $1376 \mathrm{~cm}^{-1}$ (bending vibration of $-\mathrm{CH}$ ). For the system SBR/NBR/NR a characteristic peak of the NBR phase at $2238 \mathrm{~cm}^{-1}$ (stretching vibration of $-\mathrm{C} \equiv \mathrm{N}$ ) was used for calculation of $A^{\mathrm{NBR}}$. The slope $f_{\mathrm{SBR} / \mathrm{BR}}=$ 0.33 and $f_{\mathrm{BR} / \mathrm{NR}}=0.75$ for $\mathrm{SBR} / \mathrm{BR} / \mathrm{NR}$ blend as well as $f_{\mathrm{SBR} / \mathrm{NBR}}=0.31$ and $f_{\mathrm{NBR} / \mathrm{NR}}=1.1$ for SBR/ NBR/NR blend were determined using calibrations curves [19]. Setting $L^{\mathrm{B}(\mathrm{SBR})} / L^{\mathrm{B}(\mathrm{BR})}$ and $L^{\mathrm{B}(\mathrm{BR})} / L^{\mathrm{B}(\mathrm{NR})}$ into Equations (2)-(4) the selective wetting of CNTs in ternary rubber blend can be determined.

\subsection{Structural characterization}

Optical microscopy

Optical microscopy was used to characterize the CNT macrodispersion. The ratio of the surface of non-dispersed agglomerates to that of the image $A / A_{0}$ is a measure for the filler macrodispersion. 


\section{Atomic Force Microscopy}

Investigations of filler distribution were carried out by an Atomic Force Microscope (AFM) Nanowiz$\operatorname{ard}^{\circledR}$ II (JPK-Instruments, Berlin) with a scan-head of $100 \times 100 \times 15 \mu \mathrm{m}^{3}$. For maximum resolution the $\mathrm{z}$-scanner range was reduced to $1.5 \mu \mathrm{m}$. Super sharp silicon cantilever (SSS-NCHR, Nanosensors ${ }^{\mathrm{TM}}$, with tip diameter of approx. $4 \mathrm{~nm}$ ) was used only. The AFM was operated in intermittent mode with a constant lever amplitude to keep the interaction forces in a same level to achieve a comparable phase distribution for all samples. All samples were produced by cutting in a cryo-chamber $\mathrm{CN} 30$ of a rotary microtom HM 360 (Microm) with a diamond knife at $-120^{\circ} \mathrm{C}$.

\section{FTIR spectroscopy analysis}

The analysis of the rubber-filler gel was carried out by use of a FTIR spectrometer S2000 (Perkin Elmer) equipped with a diamond single Golden Gate ATR cell (Specac). The ATR cell was pressed on a shapeless piece of rubber-filler gel with a measuring area of $2 \mathrm{~mm} \times 2 \mathrm{~mm}$. Five spectra were recorded for each sample.

\section{Results and discussion}

\subsection{Online measured electrical conductance and CNT macrodispersion}

The online conductance curves of two statistical blends CNT-SBR/BR/NR and CNT-SBR/NBR/NR without and with ethanol recorded during the mixing are presented in Figure 1. Without ethanol, CNTSBR/BR/NR and CNT-SBR/NBR/NR blends show no electrical signal (curve 1 and 2), that indicates no percolated conduction filler network formed in rubbers because of the bad filler dispersion. They show several large CNT agglomerates in optical macroscopic images with a macrodispersion $A / A_{0}$ of 15 and $35 \%$, respectively (Figure $1 \mathrm{~b}$ and $1 \mathrm{~d}$ ). When CNT/ ethanol mixture is added to SBR/BR/NR blend, a significant increase of conductance of several orders of magnitude is observed (curve 3). According to our previous work [20] the macrodispersion of filler and the online conductance correlate closely to each other. The largest change of the size of filler agglomerates, i.e. the dispersion of large filler agglomerate into smaller aggregates or even individual tubes, is determined in the period between $t_{\text {onset }}$ and $t_{\mathrm{G}_{\max }}$. Upon $t_{\mathrm{G} \text { max }}$ the online conductance decreases slightly that is related to the better distribution of small aggregates throughout the matrix and/or shortening of tubes. Thus, $t_{\mathrm{onset}}$ and $t_{\mathrm{G} \max }$ have been often used for characterization of the filler dispersion kinetics. $\mathrm{A} t_{\mathrm{G} \max }$ recorded at $5 \mathrm{~min}$ is related to a fast dispersion of CNTs in SBR/BR/NR blend. Optical microscopic image of the sample taken out at $30 \mathrm{~min}$ mixing confirms the improvement of CNT dispersion in this blend with assistance of ethanol. CNT dispersion in CNT-SBR/BR/NR blend is improved markedly with a macrodispersion reducing from 15 to $3.5 \%$ (Figure $1 \mathrm{~b}$ and $1 \mathrm{c}$ ). In contrast, ethanol shows only moderate effect on the online conductance curve and CNT dispersion of CNT-SBR/NBR/NR blend. The macrodispersion $A / A_{0}$ decreases from 35 to $20 \%$ according to Figure $1 \mathrm{~d}$ and $1 \mathrm{e}$. The reason for the insignificant effect of ethanol in the blend containing NBR may be related to the bad compatibility between ethanol and NBR. With a surface tension of $22 \mathrm{mN} / \mathrm{m}$ ethanol shows a good compatibility with the SBR, BR and NR, however, it is not compatible with the polar NBR. Upon ethanol evaporation during the mixing CNTs will be dispersed well in NBR.

\subsection{Wetting behavior of CNTs in different rubbers}

The dependence of the rubber layer $L$ on the mixing time of filled SBR, BR, NBR and NR compounds is presented in Figure 2a. With increasing mixing time the infiltration of SBR, BR, NBR and NR molecules into CNT agglomerate and the dispersion of CNTs take place simultaneously according to the infiltration model proposed by Manas-Zloczower [21]. The rubber layer $L$ of investigated rubbers without ethanol assistance increases with different rates and reaches a plateau value after $50 \mathrm{~min}$ for both SBR and BR, 10 min for NBR and $17 \mathrm{~min}$ for NR, respectively. According to our previous work [5], the development of the rubber layer $L$ can be theoretically calculated by Equation (7):

$$
L=\frac{h b t^{1 / 2}}{h b t^{1 / 2}+\frac{\rho_{\mathrm{F}}}{\rho_{\mathrm{R}}}(1-\varepsilon)}
$$

where $\varepsilon$ is the porosity and $\rho_{\mathrm{F}}$ is the density of the filler. $\rho_{\mathrm{R}}$ is the density of rubber. $b$ is a measure for wetting speed and can be described by Equation (8): 

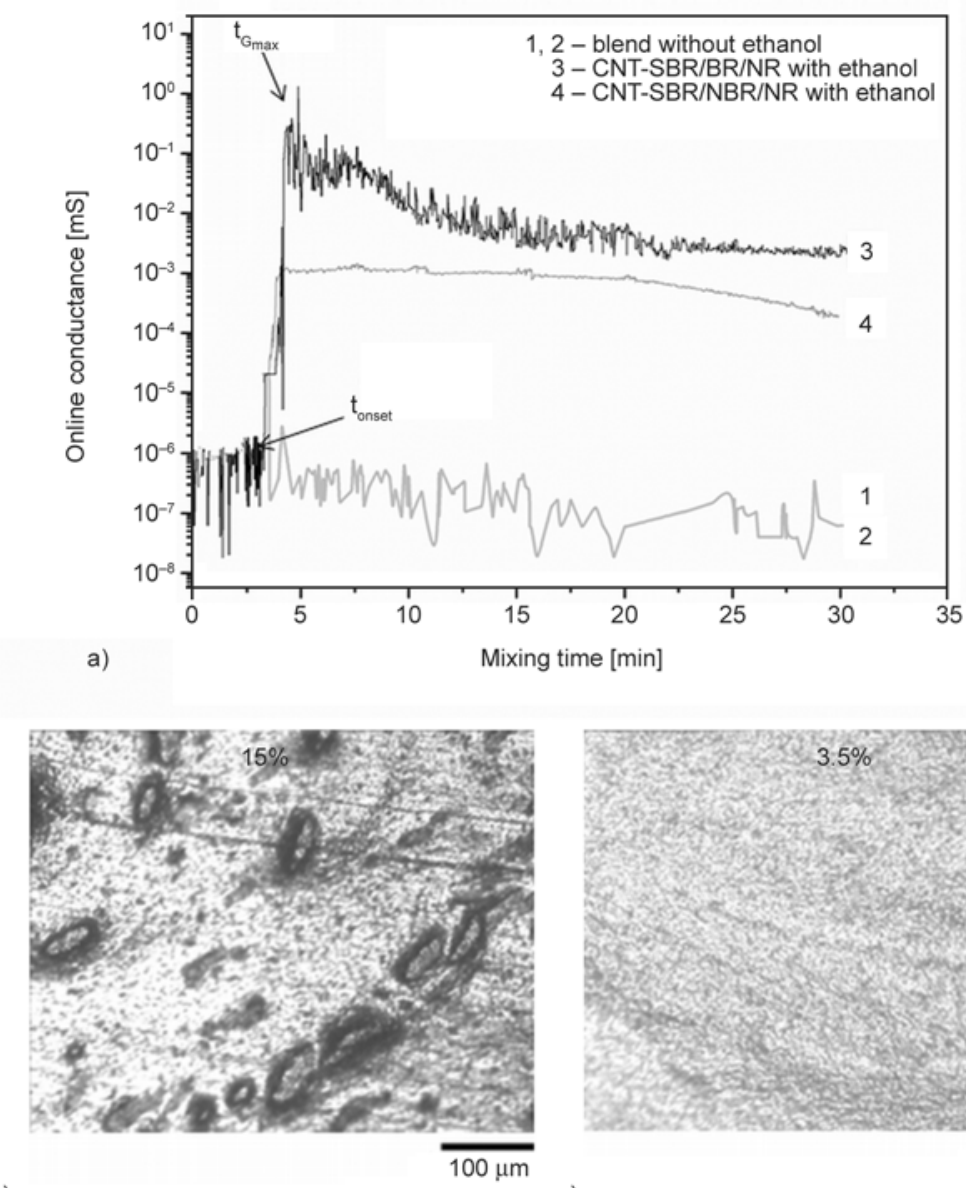

b)

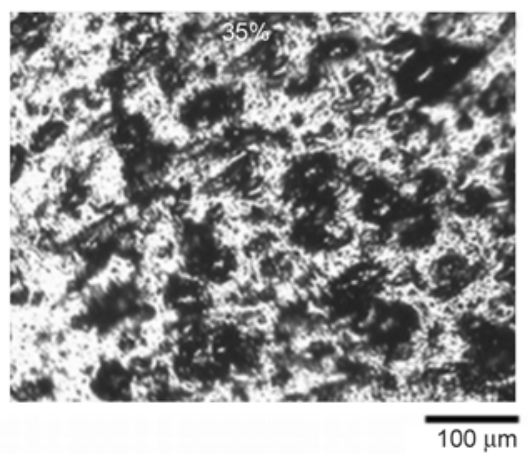

d)

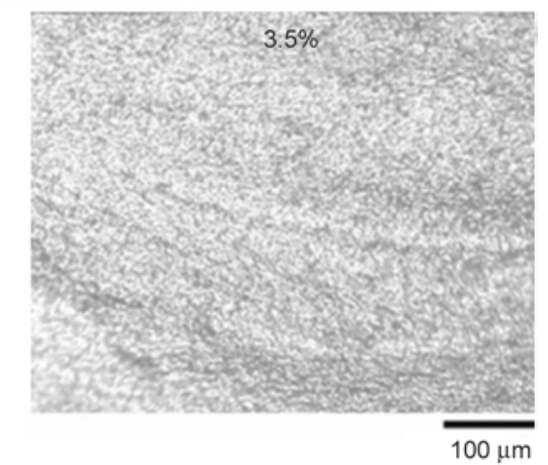

c)

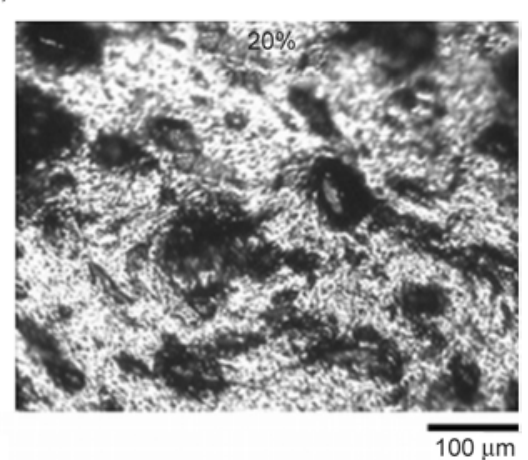

e)

Figure 1. Online conductance curves of CNT filled blends in dependence on mixing time (a) and optical microscopic images of CNT-SBR/BR/NR without (b) and with ethanol (c) as well as CNT/NBR/NR blend without (d) and with ethanol (e) taken out at 30 min mixing

$b=\frac{\varepsilon}{D_{0}}\left(\frac{S \gamma_{\mathrm{R}} \cos \theta}{3 \eta_{\mathrm{R}}}+\frac{S^{2} \Delta P}{6 \eta_{\mathrm{R}}}\right)^{1 / 2}$

In our model $S$ is considered as pore size of filler, $\Delta P$ is the pressure in the mixing chamber. $\gamma_{\mathrm{R}}$ is the surface tension of rubber, $\theta$ is the contact angle and $\eta_{\mathrm{R}}$ is the rubber viscosity. $D_{0}$ is the average agglomerate diameter. The correlation between the plateau value $L_{\mathrm{P}}$ and the factor $\mathrm{h}$ can be described by Equation (9):

$$
L_{\mathrm{P}}=\frac{\varepsilon h}{\varepsilon h+\frac{\rho_{\mathrm{F}}}{\rho_{\mathrm{R}}}(1-\varepsilon)}
$$

By fitting Equation (7) to the experimental data presented in Figure 2a with the average $\rho_{\mathrm{R}}=0.94 \mathrm{~g} / \mathrm{cm}^{3}$ and $\rho_{\mathrm{F}}=2.0 \mathrm{~g} / \mathrm{cm}^{3}, \varepsilon=0.92$ [22] the wetting speed $b^{\mathrm{SBR}}=0.19 \mathrm{~min}^{-1 / 2}$ and $b^{\mathrm{BR}}=0.19 \mathrm{~min}^{-1 / 2}$ and $b^{\mathrm{NBR}}=$ $0.47 \mathrm{~min}^{-1 / 2}$ as well as $b^{\mathrm{NR}}=0.31 \mathrm{~min}^{-1 / 2}$ can be experimentally determined for SBR, BR, NBR and NR, respectively. 

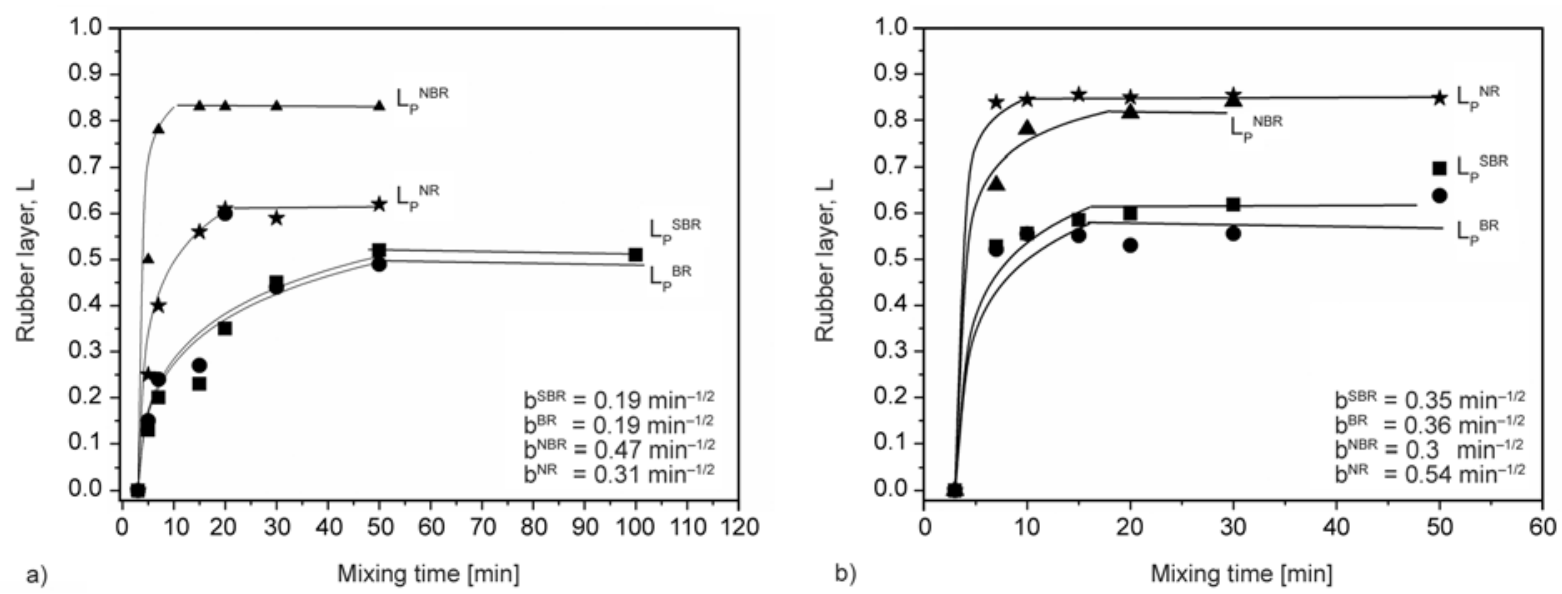

Figure 2. Development of rubber layer $L$ of SBR, BR and NBR as well as NR compound during mixing process without (a) and with (b) ethanol assistance

According to Equation (8) the wetting speed $b$ increases with increasing rubber surface tension. The high value of the wetting speed of NBR is corresponding to the high surface tension of NBR of $27 \mathrm{mN} / \mathrm{m}$ [23]. The good filler-rubber interaction of CNTs in NBR was evidenced by Verge et al. [24]. They stated that nitrile groups of NBR can be bonded with CNT surface along the mixing process through the electrostatic interactions. It comes out that the polymer bonding rate onto the CNT surface increases with the nitrile content in NBR.

Although the surface tension $\gamma_{\mathrm{NR}}=22 \mathrm{mN} / \mathrm{m}$ determined by the contact angle measurement is lower than that of SBR, NR shows a higher wetting speed $b^{\mathrm{NR}}=0.31 \mathrm{~min}^{-1 / 2}$ compared to $b^{\mathrm{SBR}}=0.19 \mathrm{~min}^{-1 / 2}$. As discussed in our previous work [20] a strong rubber-filler interaction between NR and CNTs was proposed by comparing the CNT localization in SBR/NR and SBR/isoprene (IR) blend. The solid NR was proposed to consist of $\omega$-terminal, two trans- 1,4 isoprene units, about 1000-3000 cis-1,4 isoprene units, and $\alpha$-terminal $[25,26]$. The $\omega$-terminal of the rubber molecule was postulated to be a modified dimethylallyl group linking to a functional group, which is associated with the proteins as cross-linking points in NR. On the other hand, the $\alpha$-terminal group of NR was postulated to consist of two kinds of functional group, i.e., monophosphate and diphosphate groups, which are linked with phospholipids via hydrogen bonding as a predominant linkage. When CNTs are mixed into NR the ammonium cation $\mathrm{N}^{+}$of phospholipids can interact with CNT surface via cation- $\pi$ interaction forming strong linkages beside the Van der Waals bondings. Based on the experimental result shown in Figure 2a it can be postulated that
NR can wet CNTs faster than SBR due to the cation$\pi$ interaction. Because the amount of phospholipids in NR is very small $(<1.0 \%)$ and they are associated with the chain end, the value of surface tension of NR is mainly determined by the isoprene units of the backbone. Thus, the surface tension value of NR determined by the contact angle measurement does not involve the effect of linked phospholipids and thus it cannot explain the fast wetting speed of NR vs. SBR. Based on our previous work [27], the strong interaction between NR and CNTs was quantified through the corrected surface tension of NR corr $\gamma_{\mathrm{NR}}=26 \mathrm{mN} / \mathrm{m}$, which was calculated by setting the stationary values of filler localization into the Z-model.

In Figure $2 b$, an addition of ethanol accelerates the CNT wetting by SBR, BR and NR due to the faster filler dispersion that eases the infiltration of rubber into filler agglomerate. In contrast, ethanol slows the wetting of CNTs by NBR because of the incompatibility between ethanol and NBR. The wetting speed $b^{\mathrm{SBR}}=0.35 \mathrm{~min}^{-1 / 2}$ and $b^{\mathrm{BR}}=0.36 \mathrm{~min}^{-1 / 2}$ and $b^{\mathrm{NR}}=0.54 \mathrm{~min}^{-1 / 2}$ are found to be increased, while $b^{\mathrm{NBR}}=0.3 \mathrm{~min}^{-1 / 2}$ is lower than that determined from Figure 2a.

\subsection{Selective CNT wetting in ternary blends}

On the basis of the Z-model proposed in our previous work [6] the selective wetting of filler in a ternary SBR/BR/NR blend at a thermodynamic equilibrium state can be predicted using Equations (10)-(12):

$\frac{S_{\mathrm{eq}}^{\mathrm{B}(\mathrm{BR})}}{S_{\mathrm{eq}}^{\mathrm{B}(\mathrm{SBR})}}=n_{\mathrm{BR} / \mathrm{SBR}}\left(\frac{\gamma_{\mathrm{SBR}}+\gamma_{\mathrm{F}}-2 \sqrt{\gamma_{\mathrm{SBR}} \gamma_{\mathrm{F}}}}{\gamma_{\mathrm{BR}}+\gamma_{\mathrm{F}}-2 \sqrt{\gamma_{\mathrm{BR}} \gamma_{\mathrm{F}}}}\right)^{2}$ 


$$
\begin{aligned}
& \frac{S_{\mathrm{eq}}^{\mathrm{B}(\mathrm{NR})}}{S_{\mathrm{eq}}^{\mathrm{B}(\mathrm{BR})}}=n_{\mathrm{NR} / \mathrm{BR}}\left(\frac{\gamma_{\mathrm{BR}}+\gamma_{\mathrm{F}}-2 \sqrt{\gamma_{\mathrm{BR}} \gamma_{\mathrm{F}}}}{\gamma_{\mathrm{NR}}+\gamma_{\mathrm{F}}-2 \sqrt{\gamma_{\mathrm{NR}} \gamma_{\mathrm{F}}}}\right)^{2} \\
& S^{\mathrm{B}}=S_{\mathrm{eq}}^{\mathrm{B}(\mathrm{SBR})}+S_{\mathrm{eq}}^{\mathrm{B}(\mathrm{BR})}+S_{\mathrm{eq}}^{\mathrm{B}(\mathrm{NR})}
\end{aligned}
$$

$S_{\mathrm{eq}}^{\mathrm{B}(\mathrm{SBR})}, S_{\mathrm{eq}}^{(\mathrm{BR})}$ and $S_{\mathrm{eq}}^{\mathrm{B}(\mathrm{NR})}$ are the fractions of filler surface wetted by rubber component of the ternary blend at an equilibrium state. $n_{\mathrm{BR} / \mathrm{SBR}}$ and $n_{\mathrm{NR} / \mathrm{BR}}$ are the mass ratio of the rubber phase BR to SBR and NR to BR, respectively. $\gamma_{\mathrm{SBR}}, \gamma_{\mathrm{BR}}, \gamma_{\mathrm{NR}}$ and $\gamma_{\mathrm{F}}$ are the surface tension values of the blend components and filler, respectively. $S^{\mathrm{B}}$ is the total surface of filler added to the blend. Setting the surface tension values $\gamma_{\mathrm{SBR}}=23.8 \mathrm{mN} / \mathrm{m}, \gamma_{\mathrm{BR}}=22 \mathrm{mN} / \mathrm{m}$ and corr $\gamma_{\mathrm{NR}}=$ $26 \mathrm{mN} / \mathrm{m}$ of rubber components into Equations (10)(12) with $n_{\mathrm{BR} / \mathrm{SBR}}=1$ and $n_{\mathrm{NR} / \mathrm{BR}}=1$ for the investigated blends, three Z-shaped master curves demonstrating the selective wetting of CNTs by three blend components in dependence on the filler surface tension can be created as seen in Figure 3a. Fitting the
CNT surface tension $\gamma_{\mathrm{F}}=30 \mathrm{mN} / \mathrm{m}$ [22] into the master curves a surface fraction wetted by each phase, $S_{\mathrm{eq}}^{\mathrm{B}(\mathrm{SBR})}=0.13, S_{\mathrm{eq}}^{\mathrm{B}(\mathrm{BR})}=0.07$ and $S_{\mathrm{eq}}^{\mathrm{B}(\mathrm{NR})}=0.8$, respectively, can be predicted for a thermodynamic equilibrium state. For $\mathrm{SBR} / \mathrm{NBR} / \mathrm{NR}$ blend $S_{\mathrm{eq}}^{\mathrm{B}(\mathrm{SBR})}==0.02, S_{\mathrm{eq}}^{\mathrm{B}(\mathrm{NBR})}=0.65$ and $S_{\mathrm{eq}}^{\mathrm{B}(\mathrm{NR})}=0.33$ are theoretically determined from Figure $3 \mathrm{~b}$.

The CNT selective wetting determined can be qualitatively demonstrated by TGA and FTIR investigations of the rubber-filler gels. In Figure 4 the TGA curves of rubber-filler gel of CNT-SBR/BR/NR and CNT-SBR/NBR/NR blends taken out at 50 min mixing are presented. The onset of degradation temperature of NR is about $370^{\circ} \mathrm{C}$, while SBR, BR and NBR start to be degraded at $430^{\circ} \mathrm{C}$. Making use the different degradation temperature of NR from the other blend partners the contribution of NR is determined by means of differential thermogravimetric analysis and shown in Figure 4a. It is obvious that the contribution of the wetted NR in the rubber-filler gel of CNT-SBR/BR/NR blend is three times higher than
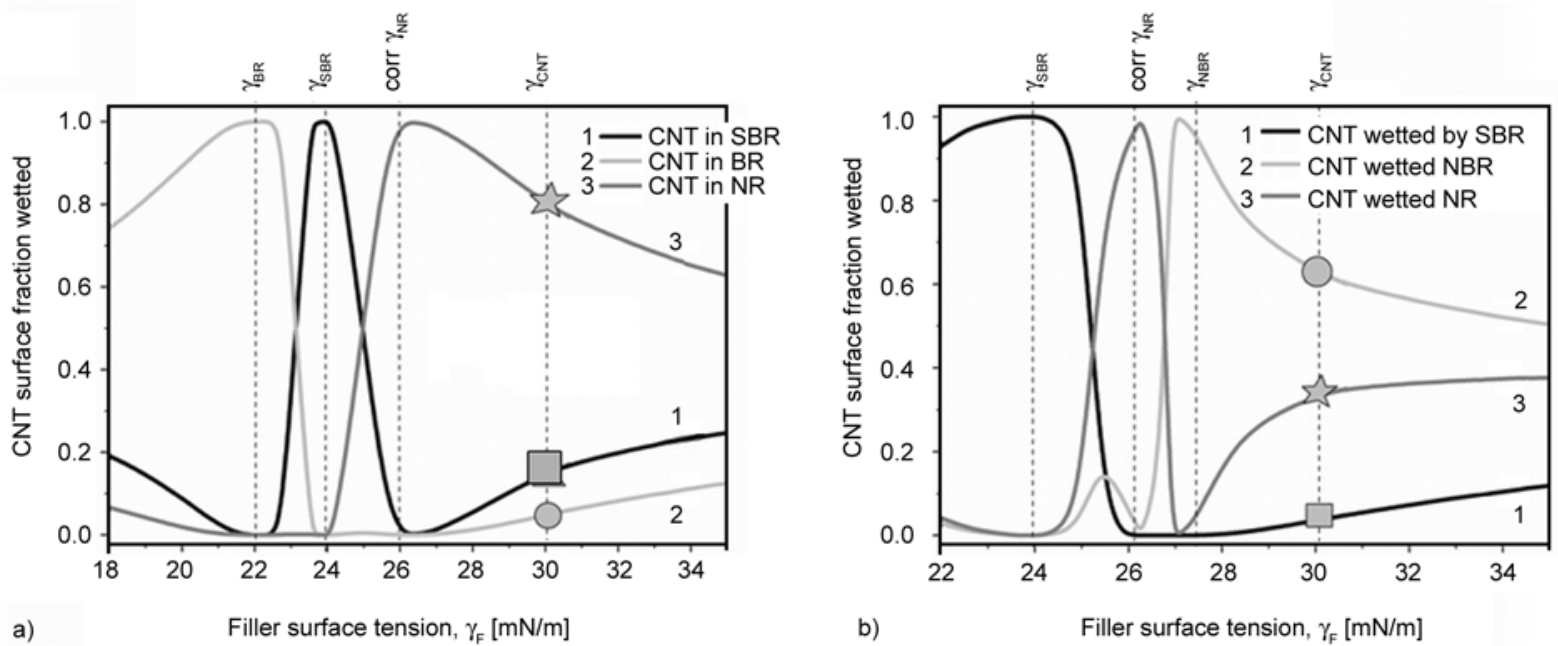

Figure 3. Master curves of selective filler wetting in 33/33/34 SBR/BR/NR (a) and 33/33/34 SBR/NBR/NR (b) blend deter-

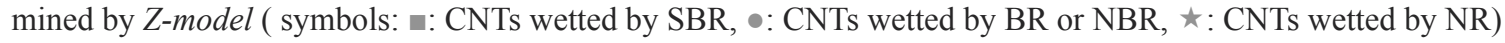
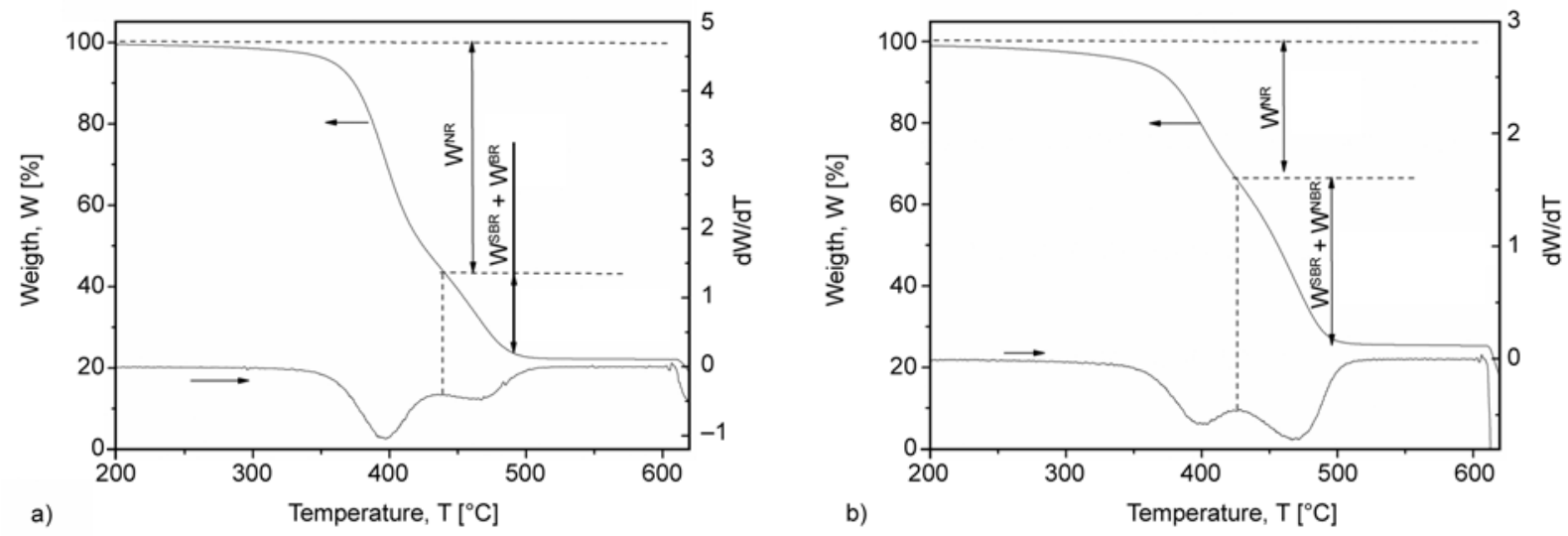

Figure 4. TGA curves of blend and its rubber-filler gel of CNT-SBR/BR/NR (a) and CNT-SBR/NBR/NR (b), respectively 

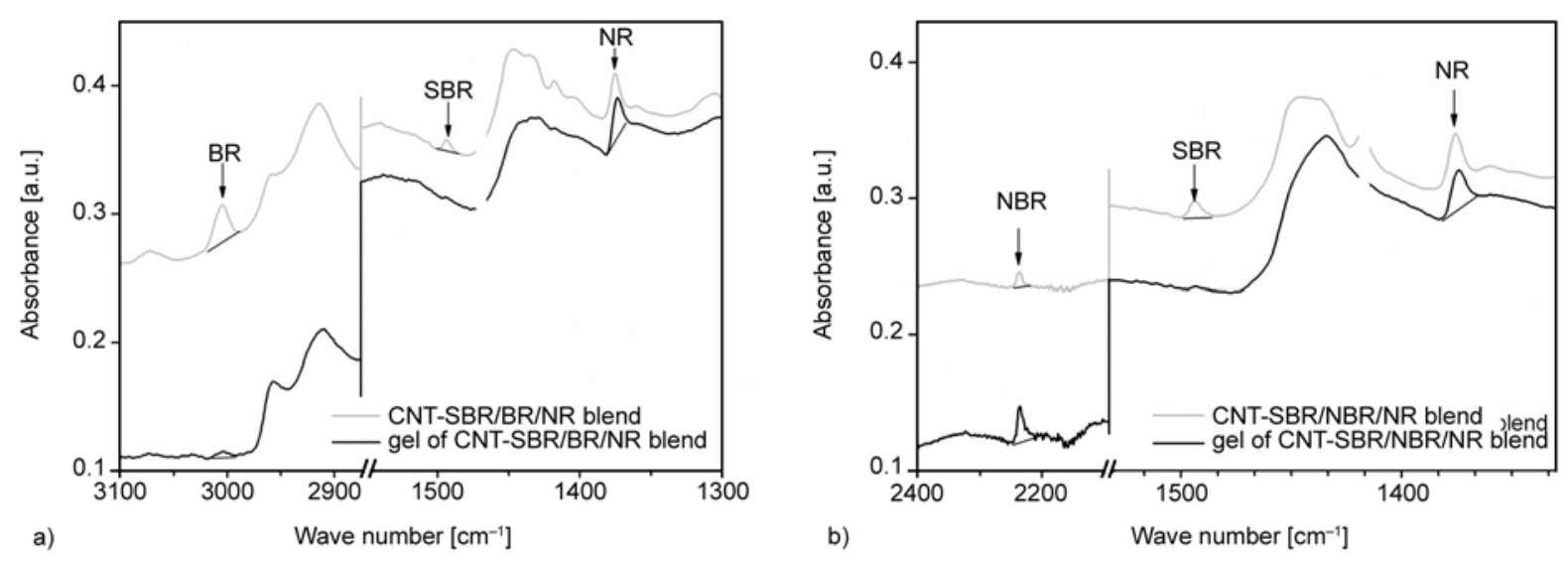

Figure 5. FTIR spectra of blend and its rubber-filler gel of CNT-SBR/BR/NR (a) and CNT-SBR/NBR/NR (b), respectively

that of SBR and BR together (Figure 4a) and it is only one third of the total wetting rubbers in the gel of CNT-SBR/NBR/NR blend (Figure 4b).

FTIR spectra of both blends and their rubber-filler gel are shown in Figure 5a and 5b. Characteristic peaks for SBR, NBR and NR as well as BR, are clearly detected in the spectra. A characteristic peak of SBR at $1494 \mathrm{~cm}^{-1}$ (stretching vibration of the phenyl ring) and NBR at $2238 \mathrm{~cm}^{-1}$ (stretching vibration of $-\mathrm{C} \equiv \mathrm{N}$ ) and $\mathrm{NR}$ at $1376 \mathrm{~cm}^{-1}$ (bending vibration of $-\mathrm{CH}$ ) as well as BR at $3004 \mathrm{~cm}^{-1}$ (stretching vibration $\mathrm{C}-\mathrm{H}$ ) are used for evaluation of the rubber-filler gel composition. The spectral of the blend and its gel were normalized with respect to the NR peak intensity for comparison purpose. It is clearly observed for CNT-SBR/BR/NR blend that upon extraction the intensity of bound SBR and BR of the gel contributes only $10 \%$ of that of the blend (Figure $5 \mathrm{a}$ ). It indicates that CNTs are mainly wetted by the NR phase. Comparing the spectral of CNT-SBR/NBR/ NR blend with that of its gel in Figure 5b we observe that the intensity of NBR increases twice after extrac-

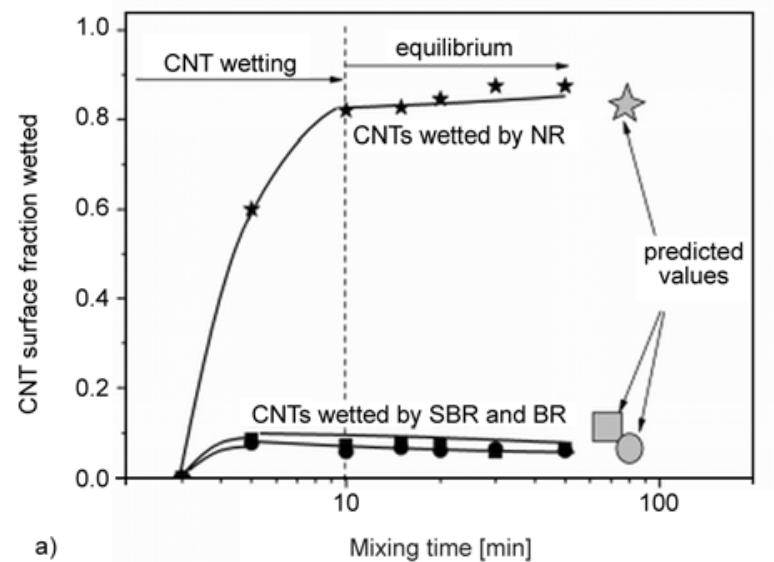

tion experiment, while the SBR peak nearly disappears. Thus, in this blend CNTs are significantly wetted by the NBR phase and only moderately by NR phase. Similarly to the CNR-SBR/BR/NR blend the SBR phase in CNT-SBR/NBR/NR blend seems not to wet CNTs.

The kinetics of CNT wetting in CNT-SBR/BR/NR blend in presence of ethanol was quantified by the wetting concept. As seen in Figure 6a the CNT surface is wetted differently after adding 5 phr CNT into the blend. It is clear that NR wets more CNT surface than SBR and BR in the first mixing stage (up to $10 \mathrm{~min}$ ) due to the higher wetting speed as observed in Figure $2 \mathrm{a}$ as well as the strong cation- $\pi$ interaction between NR and CNTs as discussed in [20], while CNTs are insignificantly wetted by SBR and BR phase. A CNT surface fraction of about 0.81 wetted by NR phase was found at $10 \mathrm{~min}$. Both SBR and BR wet only a surface fraction of 0.19 . In the second stage from 10 to $50 \mathrm{~min}$, no change in the wetting kinetics of CNTs was observed, i.e. the wetting reaches its thermodynamic equilibrium state.

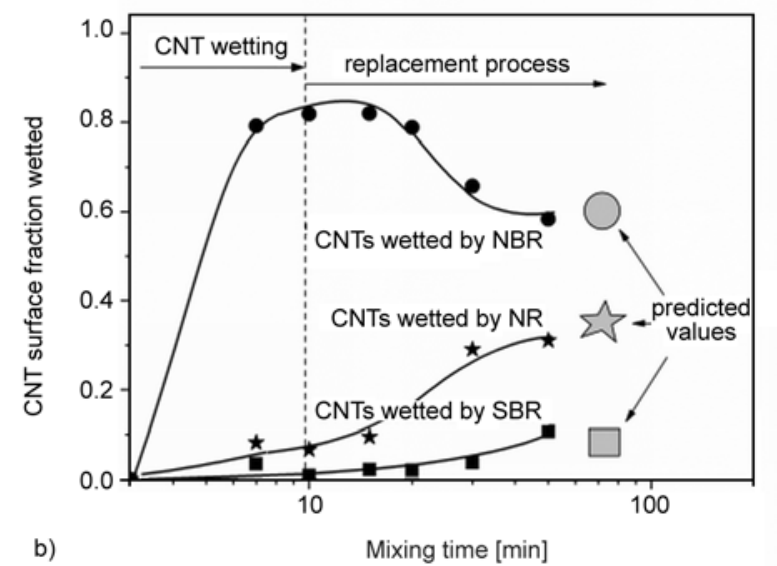

Figure 6. Kinetics of selective filler wetting in statistical blends 33/33/34 CNT-SBR/BR/NR (a) and 33/33/34 CNT$\mathrm{SBR} / \mathrm{NBR} / \mathrm{NR}$ (b) obtained by the wetting concept. Values predicted by the Z-model are displayed (grey symbols) 
By replacing the non-polar BR component by the polar NBR the kinetics of CNT wetting in CNT$\mathrm{SBR} / \mathrm{NBR} / \mathrm{NR}$ blend is characterized and presented in Figure 6b. In the first mixing stage up to $10 \mathrm{~min}$, CNTs are wetted very fast by NBR, while NR and SBR wet CNTs much slower. The fast wetting of CNTs by NBR is clearly explained by the high wetting speed $b^{\mathrm{NBR}}$ determined from Figure 2. A CNT surface fraction of about 0.82 was determined at $10 \mathrm{~min}$, when the wetting process ends. In the second stage from 10 to $50 \mathrm{~min}$, a replacement process of NBR chains by NR chains took place. A portion of bound NBR chains was replaced by NR chains on the CNT surface. Only a minor surface of CNTs is wetted by the SBR phase after long mixing time. At 50 min mixing time CNT surafce fraction wetted by each blend phase, $S^{\mathrm{B}(\mathrm{SBR})}=0.1, S^{\mathrm{B}(\mathrm{NBR})}=0.6$ and $S^{\mathrm{B}(\mathrm{NR})}=0.3$ are observed. These values are corresponding well to the predicted ones shown in Figure $3 \mathrm{~b}$. It comes out that the electrostatic interaction between nitrile groups of NBR with CNTs is stronger than the cation- $\pi$ interaction between NR and CNTs. The quantitative characterization of CNT wetting in both blends can be visualized well by AFM. In Figure 7a AFM images of CNT-SBR/BR/NR blend made by two magnifications show obviously that CNTs are encapsulated by a dark rubber phase. By taking into consideration the difference hardness of blend components the dark phase is identified as NR. SBR and BR are compatible and form a continuous phase, which seems not to wet CNTs. The morphology of CNT-SBR/NBR/NR blend presented in Figure $7 \mathrm{~b}$ shows some unfilled domains, which can be attributed to the SBR phase. A large amount of CNTs (bright tubes) is found in the NBR continuous phase (gray continuous phase), which is surrounding the SBR and NR domains. For the CNT-SBR/NBR/NR blend, the presence of ethanol accelerates the dispersion of CNTs localized in SBR and NR phase but at the same time slowdowns the CNT dispersion in NBR phase. According to Figure 6b NBR hosts $60 \mathrm{wt} \%$ of CNTs used and, thus, that results in the bad dispersion of CNTs in the whole blend.

In order to investigate the replacement process of one rubber component by the other ones (desorption and adsorption) taking place on the CNT surface during masterbatch mixing, the kinetics of CNT wetting of (CNT-SBR)/BR/NR blend prepared by blending the CNT-SBR masterbatch with the other fresh rubbers, BR and NR, was determined and is presented in Figure 8a. It is found that SBR chains prebound to the CNT surface from masterbatch mixing is strongly replaced by NR and slightly by BR during the subsequent mixing process. The replacement process took place within about $5 \mathrm{~min}$. Afterwards the wetting of CNTs seems to reach the stationary state
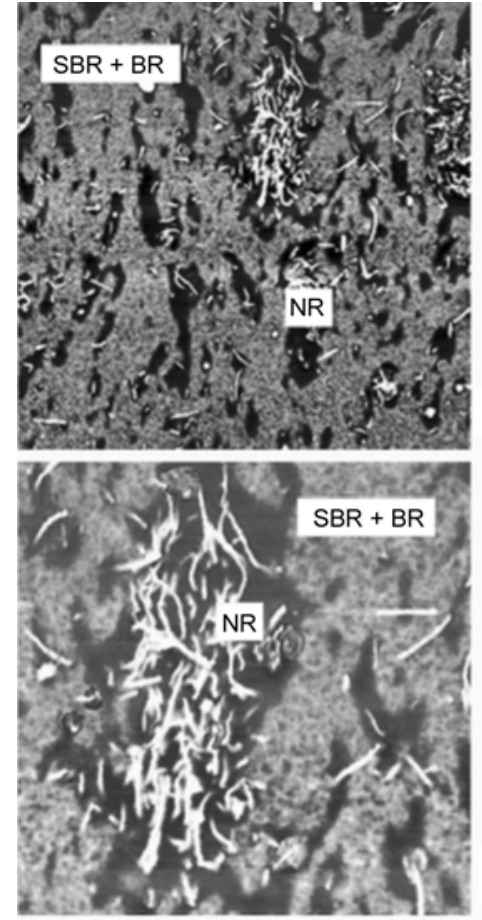

a)

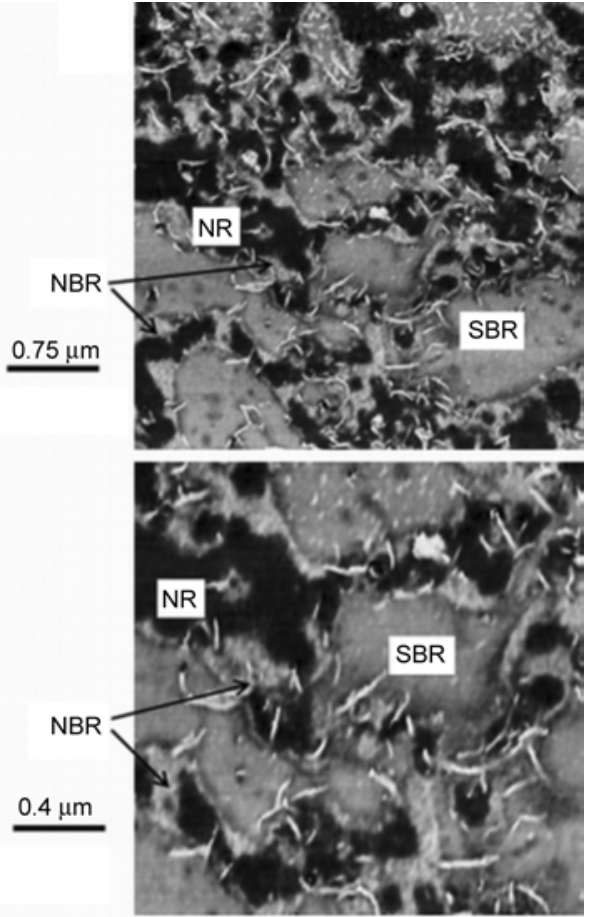

b)

Figure 7. AFM images of CNT-SBR/BR/NR (a) and CNT-SBR/NBR/NR blend (b) at two magnifications. 

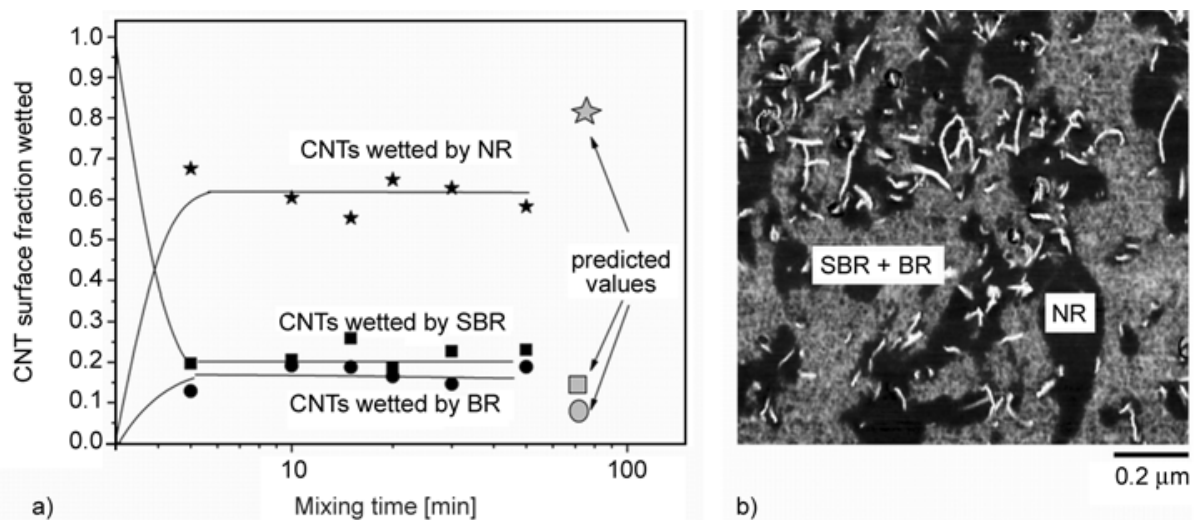

b)
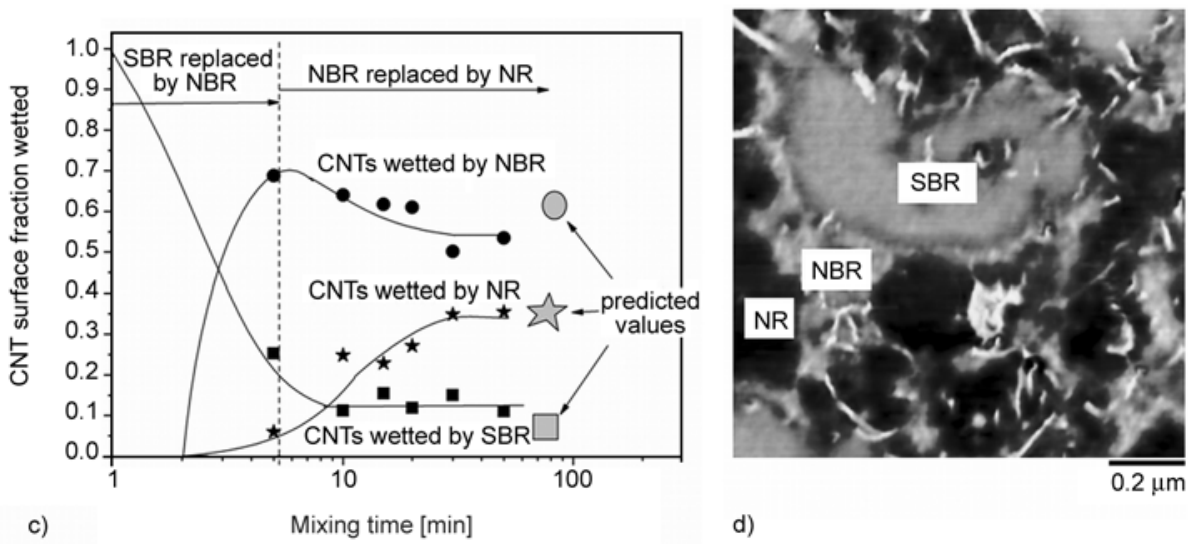

d)

Figure 8. Kinetics of CNT selective wetting in (SBR-CNT)/BR/NR blend (a) and (CNT-SBR)/NBR/NR blend (c) as well as AFM images of samples taken out at 50 min mixing $(b, d)$

of CNT wetting, which is corresponding well to that of statistical blend and to the prediction. A closer look at the result from Figure 8a reveals that the experimentally determined values of CNT surface fraction wetted by SBR phase remains slightly higher than that predicted. The reason may be related to the fact that during the masterbatch mixing of CNTSBR some radicals of SBR are formed and they can chemically interact with CNTs. Such chemical bondings are stable and cannot be replaced by NR. It is clear from AFM image of the (CNT-SBR)/BR/NR blend shown in Figure $8 \mathrm{~b}$ that CNTs (bright tubes) are mainly wetted by the NR phase (dark domains). SBR and BR are compatible, thus they form a continuous phase (gray phase). Some individual tubes are observed in this matrix.

The effect of rubber polarity and blend morphology on the kinetics of selective wetting of CNTs can be observed in the case of (CNT-SBR)/NBR/NR blend. It is clear from Figure 8c that the pre-bound SBR chains are firstly replaced only by NBR in the first mixing stage up to $7 \mathrm{~min}$, while NR seems not to take part in the replacement process. Afterwards, the CNT surface fraction wetted by NBR decreases, while the surface fraction wetted by NR increases, i.e. the bound NBR molecules are replaced by the free NR molecules. This process takes place until the predicted thermodynamic equilibrium state is reached. The obtained progression of selective wetting of CNTs is clearly understood by examining AFM images shown in Figure 8d. The SBR and NR domains are surrounded by a layer of NBR, i.e SBR has no direct contact with NR. Therefore, in the first period of mixing CNT-SBR masterbatch with NBR and NR, only NBR gets into contact with CNT-SBR masterbatch and replaces the bound SBR. At a mixing time of $15 \mathrm{~min}$ NBR wets the most surface of CNTs. According to the calculation by means of $Z$ model for the binary 50/50 SBR/NBR blend CNTs are entirely wetted by NBR at an equilibrium state (the result is not shown in the present work). However, according to the Z-model for 50/50 NBR/NR blend a CNT surface fraction of 0.6 is wetted by NBR and 0.4 by NR at an equilibrium state. Thus, after 15 min mixing the freshly bound NBR is replaced by NR until the equilibrium state is reached as seen in Figure 8c. 


\section{Conclusions}

It was proposed that phospholipids, which are linked to the $\alpha$-terminal of NR can interact with the CNT surface through cation- $\pi$ interactions forming strong bonding between NR and CNTs. These strong interactions are the main reason for the favorable wetting of CNT by the NR phase in SBR/BR/NR blend. Using the corrected surface tension value of NR, which involves the effect of phospholipids found in our previous work the selective wetting of CNTs in ternary rubber blends can be well predicted for a thermodynamic equilibrium state. By replacing the non-polar BR by a polar rubber like NBR as a blend component CNTs are wetted by NBR slightly more than by NR thanks to the strong interaction between CNTs and nitrile groups of NBR. SBR remains unbound to CNTs in both blends. The sequence of replacement of the rubber molecules pre-bound to CNT surface by the other ones, for instance SBR first by NBR and then NBR by NR, is well explained by taking into consideration the blend morphology made by AFM.

\section{Acknowledgements}

The authors wish to thank the Deutsche Forschungsgemeinschaft (DFG) (Project Nr. LE 3202/1-1) and Vietnam National Foundation for Science and Technology Development (Nafosted) (Grant number 104.02-2014.90) for the financial support.

\section{References}

[1] Bokobza L.: Multiwall carbon nanotube elastomeric composites: A review. Polymer, 48, 4907-4920 (2007). DOI: $10.1016 /$ j.polymer.2007.06.046

[2] Rahmat M., Hubert P.: Carbon nanotube-polymer interactions in nanocomposites: A review. Composites Science and Technology, 72, 72-84 (2011). DOI: $10.1016 /$ j.compscitech.2011.10.002

[3] McNally T., Pötschke P.: Polymer-carbon nanotube composites: Preparation, properties and applications, Woodhead, Cambridge (2011).

[4] Alig I., Pötschke P., Lellinger D., Skipa T., Pegel S., Kasaliwal G. R., Villmow T.: Establishment, morphology and properties of carbon nanotube networks in polymer melts. Polymer, 53, 4-28 (2012). DOI: $10.1016 /$ j.polymer.2011.10.063
[5] Le H. H., Keller M., Hristov M., Ilisch S., Xuan T. H., Do Q. K., Pham T., Stöckelhuber K-W., Heinrich G., Radusch H-J.: Selective wetting and localization of silica in binary and ternary blends based on styrene butadiene rubber, butadiene rubber, and natural rubber. Macromolecular Materials and Engineering, 298, 10851099 (2013). DOI: $10.1002 /$ mame.201200307

[6] Le H. H., Ilisch S., Heinrich G., Radusch H-J.: Filler migration in natural rubber blends during the mixing process. in 'Natural rubber materials: Volume 1: Blends and IPNs' (Eds: Thomas S., Han C. C., Pothen L., Rajisha K. R., Maria H.) Royal Society of Chemistry, Cambridge, 132-176 (2014). DOI: $10.1039 / 9781849737647-00132$

[7] Jiang H-X., Ni Q-Q., Natsuki T.: Design and evaluation of the interface between carbon nanotubes and natural rubber. Polymer Composites, 32, 236-242 (2011). DOI: $10.1002 /$ pc. 21040

[8] Jiang M-J., Dang Z-M., Yao S-H., Bai J.: Effects of surface modification of carbon nanotubes on the microstructure and electrical properties of carbon nanotubes/ rubber nanocomposites. Chemical Physics Letters, 457, 352-356 (2008).

DOI: $10.1016 /$ j.cplett.2008.04.022

[9] Frogley M. D., Ravich D., Wagner H. D.: Mechanical properties of carbon nanoparticle-reinforced elastomers. Composites Science and Technology, 63, 1647-1654 (2003).

DOI: $10.1016 / \mathrm{S} 0266-3538(03) 00066-6$

[10] Bokobza L.: A Raman investigation of carbon nanotubes embedded in a soft polymeric matrix. Journal of Inorganic and Organometallic Polymers and Materials, 22, 629-635 (2012). DOI: $10.1007 / \mathrm{s} 10904-011-9590-7$

[11] Das A., Stöckelhuber K. W., Jurk R., Saphiannikova M., Fritzsche J., Lorenz H., Klüppel M., Heinrich G.: Modified and unmodified multiwalled carbon nanotubes in high performance solution-styrene-butadiene and butadiene rubber blends. Polymer, 49, 5276-5283 (2008). DOI: $10.1016 /$ j.polymer.2008.09.031

[12] Choi S-S.: Difference in bound rubber formation of silica and carbon black with styrene-butadiene rubber. Polymers for Advanced Technologies, 13, 466-474 (2002).

DOI: $10.1002 /$ pat.211

[13] Kralevich M. L., Koening J. L.: FTIR analysis of silicafilled natural rubber. Rubber Chemistry and Technology, 71, 300-309 (1998). DOI: $10.5254 / 1.3538486$

[14] Choi S-S.: Characterization of bound rubber of filled styrene-butadiene rubber compounds using pyrolysisgas chromatography. Journal of Analytical and Applied Pyrolysis, 55, 161-170 (2000). DOI: 10.1016/S0165-2370(99)00095-9 
[15] Sierra L., López B. L., Guth J. L.: Microstructural studies of the interactions in SB rubber and mesoporous silica mixtures. Materials Research Innovations, 5, 268-276 (2000).

DOI: $10.1007 / \mathrm{s} 10019-002-0160-\mathrm{Z}$

[16] Serizawa H., Ito M., Kanamoto T., Tanaka K., Nomura A.: Structural changes during mechanical mixing in carbon black-natural rubber systems studied by pulsed NMR. Polymer Journal, 14, 149-154 (1982).

DOI: $10.1295 /$ polymj.14.149

[17] Ono S., Kiuchi Y., Sawanobori J., Ito M.: Structure development in silica-filled rubber composites. Polymer International, 48, 1035-1041 (1999).

DOI: $10.1002 /($ SICI) 1097-0126(199910)48:10<1035::

$$
\text { AID-PI265>3.0.CO;2-5 }
$$

[18] Le H. H., Ilisch S., Kasaliwal G. R., Radusch H-J.: Filler phase distribution in rubber blends characterized by thermogravimetric analysis of the rubber-filler gel. Rubber Chemistry and Technology, 81, 767-781 (2008). DOI: $10.5254 / 1.3548231$

[19] Le H. H., Ilisch S., Heidenreich D., Wutzler A., Radusch H-J.: Kinetics of the phase selective localization of silica in rubber blends. Polymer Composites, 31, 1701-1711 (2010). DOI: $10.1002 /$ pc. 20960

[20] Le H. H., Parsekar M., Ilisch S., Henning S., Das A., Stöckelhuber K-W., Beiner M., Ho C. A., Adhikari R., Wießner S., Heinrich G., Radusch H-J.: Effect of nonrubber components of NR on the carbon nanotube (CNT) localization in SBR/NR blends. Macromolecular Materials and Engineering, 299, 569-582 (2014). DOI: 10.1002/mame.201300254

[21] Manas-Zloczower I.: Analysis of mixing in polymer processing equipment. Rheology Bulletin, 66, 5-8 (1997).
[22] Puretzky A. A., Geohegan D. B., Jesse S., Ivanov I. N., Eres G.: In situ measurements and modeling of carbon nanotube array growth kinetics during chemical vapor deposition. Applied Physics A, 81, 223-240 (2005). DOI: $10.1007 / \mathrm{s} 00339-005-3256-7$

[23] Stöckelhuber K-W., Das A., Jurk R., Heinrich G.: Contribution of physico-chemical properties of interfaces on dispersibility, adhesion and flocculation of filler particles in rubber. Polymer, 51, 1954-1963 (2010). DOI: $10.1016 /$ j.polymer.2010.03.013

[24] Verge P., Peeterbroeck S., Bonnaud L., Dubois P.: Investigation on the dispersion of carbon nanotubes in nitrile butadiene rubber: Role of polymer-to-filler grafting reaction. Composites Science and Technology, 70, 1453-1459 (2010).

DOI: 10.1016/j.compscitech.2010.04.022

[25] Tarachiwin L., Sakdapipanich J., Ute K., Kitayama T., Tanaka Y.: Structural characterization of $\alpha$-terminal group of natural rubber. 2. Decomposition of branchpoints by phospholipase and chemical treatments. Biomacromolecules, 6, 1858-1863 (2005). DOI: $10.1021 / \mathrm{bm} 058004 \mathrm{p}$

[26] Tanaka Y., Mori M., Ute K., Hatada K.: Structure and biosynthesis mechanism of rubber from fungi. Rubber Chemistry and Technology, 63, 1-7 (1990). DOI: $10.5254 / 1.3538238$

[27] Le H. H., Abhijeet S., Ilisch S., Klehm J., Henning S., Beiner M., Sarkawi S. S., Dierkes W., Das A., Fischer D., Stöckelhuber K-W., Wiessner S., Khatiwada S. P., Adhikari R., Pham T., Heinrich G., Radusch H-J.: The role of linked phospholipids in the rubber-filler interaction in carbon nanotube (CNT) filled natural rubber (NR) composites. Polymer, 55, 4738-4747 (2014). DOI: $10.1016 /$ j.polymer.2014.07.043 